\title{
Reverse Shoulder Arthroplasty - A Literature Review
}

\author{
Aatif Mahmood, Joby Jacob George Malal ${ }^{*}$ and Mohammed Waseem
}

\author{
Macclesfield District General Hospital, Victoria Road, Macclesfield, Cheshire, SK10 3BL, UK
}

\begin{abstract}
Professor Grammont revolutionised shoulder surgery with his reverse shoulder arthroplasty design. Patients who had poor results from a conventional shoulder replacement because of cuff deficiency can now be treated effectively. Although designed for cuff tear arthropathy, indications continue to evolve and broaden. The initial results look very promising and the implant has gained much popularity over the years. The article provides an extensive literature review of the indications, results and complications for reverse shoulder arthroplasty.
\end{abstract}

Keywords: Cuff deficiency, shoulder arthritis, shoulder arthroplasty, reverse shoulder arthroplasty, rotator cuff arthropathy.

\section{INTRODUCTION}

Total shoulder arthroplasty provides significant pain relief and improves shoulder movements, where the joint is damaged by arthritis, infection or trauma. These implants rely on a functioning rotator cuff which helps stabilize the joint and restore shoulder function. However, unpredictable results are seen in patients in whom the rotator cuff is torn [1-4]. This has led to the birth of the reverse shoulder prosthesis.

\section{HISTORY AND BIOMECHANICS}

Rotator cuff muscles encircle the humeral head, and compress it against the glenoid, thus providing a fulcrum on which the deltoid can lever to elevate the arm. When the function of the rotator cuff is lost the humeral head displaces superiorly, with loss of a functioning fulcrum. Contraction of the deltoid is unable to raise the arm as the head does not rotate on the glenoid. The displacement of the humeral head towards the acromion and coracoacromial arch can lead to a painful acromial erosions and glenohumeral arthritis. Total shoulder replacement in these cuff deficient cases is associated with a high rate of failure because of a "rocking horse" phenomenon which leads to eccentric glenoid loading and failure [5]. Consequently, hemiarthroplasty was the most appropriate treatment option for patients with arthritis secondary to a rotator cuff deficiency. Although pain improved, there was limited improvement in function and the results were compromised by glenoid and acromial bone erosion [6,7]. To prevent the proximal humeral migration, constrained and semi constrained implants were tried but all of these failed because of excessive stress on the constraints causing implant loosening [8]. To compensate for the rotator cuff deficiency, the ball and socket articulations were reversed. A number of implants based on this idea were introduced - Fenlin [9], Kessel [10], Gerard [11] and Kolbel [12] to name a few. Unfortunately, most of these remained

\footnotetext{
*Address correspondence to this author at the Department of Orthopaedics, Macclesfield District General Hospital, Victoria Road, Macclesfield, Cheshire, SK10 3BL, UK; Tel: +44 1625 661315; Fax: +44 1625 425873;
} E-mail: jjgeorgemalal@gmail.com experimental while others showed loosening of the glenoid component at follow-up and were therefore abandoned. Brostrom et al. [10] reported poor mean active elevation, a high reoperation rate and a high incidence of loosening for the Kessel prostheses followed up for 5 years. Wretenberg and Wallensten [13] similarly noted early glenoid loosening. Another common flaw in these early designs was a glenoid fixation that extended into a laterally projecting neck, which then extended in to a lateralised, spherical glenoid component. These prostheses failed because of excessive torque and shear forces at the glenoid component-bone interface [14].

In 1987, Professor Paul Grammont presented a new concept of reversed total shoulder arthroplasty [15]. His revolutionary design was based on 4 key principles: (a) intrinsic prosthetic stability. (b) Concavity of the supporting part and convexity of the weight bearing part (glenoid). (c) Glenosphere centre at or within the glenoid neck. (d) Medialised and distalised centre of rotation.

Conventional total shoulder arthroplasty combines a large prosthetic head with a shallow glenoid component, and to avoid glenohumeral dislocation the joint reaction force vector must be within $30^{\circ}$ of the centre line $[16,17]$. This is easily achieved when the rotator cuff and deltoid muscle are working in coordination. When the rotator cuff is deficient, the dominant deltoid contraction creates a proximally directed force vector causing the head to dislocate rather than abduct. In the reverse shoulder design the risk of dislocation is reduced as the humeral concave component is larger and deeper than in TSA and it articulates with a large hemispheric ball. Furthermore, a non-anatomical head-neckshaft angle of $155^{\circ}$ adds more stability. Thus, the angle that the force vector can subtend without risk of dislocation is increased to $45^{\circ}[16,17]$. Contraction of the deltoid does not cause superior humeral migration but causes rotation about a medialised centre of rotation which produces abduction [17]. Before the advent of reverse shoulder arthroplasty, hooded glenoids were tried but a high failure rate was noted $[18,19]$. This led surgeons to conclude that superior migration of the humeral head can be compensated by reversing the joint i.e. with a convex glenoid and concave humerus $[5,20]$. In designing his revolutionary prosthesis Grammont removed 
the neck, thus medialising the centre of rotation at the former glenoid surface. This led to increased compressive forces passing through the prosthesis-bone interface and reduced shearing forces, preventing loosening of the glenoid component. However, this new design caused another problem. When the arm was in adduction the humeral head caused impingement on the scapular neck leading to erosion of the bony glenoid known as inferior scapular notching. It remains to be seen whether this is of any clinical significance. Medialising the centre of rotation employs more deltoid fibres for elevation and distalising it increases the tension on the deltoid, resulting in a more powerful deltoid contraction. This leads to deltoid compensating for the lack of a rotator cuff [14].

\section{INDICATIONS}

Cuff tear arthropathy -massive cuff tear, superior migration of the humeral head with glenohumeral arthritis was the original indication for reverse shoulder arthroplasty [21-23]. However, over time the indications have expanded and now include inflammatory arthritis with associated rotator cuff tears [24], acute fractures [23, 25], fracture sequelae $[23,25,26]$, reconstruction after tumour resection $[23,27,28]$, revision arthroplasty with cuff deficiency $[23,26,29,30]$ and pseudoparalysis without arthritis [23, 31].

It should be determined pre-operatively that the glenoid has adequate bone stock to achieve stable base plate fixation. In cases of revision surgery, it is important to ascertain this on preoperative CT or MRI scan of the glenoid. A functioning deltoid is also required for forward elevation and abduction. The implant increases the moment arms of the anterior and the middle deltoid and loss of abduction is noticed in anterior deltoid insufficiency [32, 33]. Axillary nerve palsy is a contraindication as there is a high risk of instability with a non functioning deltoid muscle [17]. Further contraindications include infection, neuroarthropathy and glenoid bone defects. Progressive deterioration of functional results is to be expected after 6 years [20,34] and hence reverse shoulder replacement is reserved for elderly patients with low functional demands.

\section{RESULTS}

The results of reverse shoulder arthroplasty depend on the aetiology. Additionally, the complications and reoperation rates differ for primary and revision reverse shoulder arthroplasty [20,23,34,35]. A severe limitation to the studies with larger numbers in the literature is that they include a mixture of underlying diagnosis. Wall et al. [23] reviewed the results of 191 retained reverse shoulder arthroplasties according to aetiology and found that patients with primary cuff tear arthropathy, primary osteoarthritis with a rotator cuff tear and a massive rotator cuff tear without arthritis had the best outcome. There was no significant difference between the three groups in terms of Constant scores, range of motion and subjective ratings. Other studies [26,34] also demonstrated that cuff tear arthropathy and massive cuff tear are the most suitable indications for RSA. Table $\mathbf{1}$ gives a summary of all previous studies in the literature with short to medium term results.

\section{Cuff Tear Arthropathy (CTA) and Massive Rotator Cuff Tears (MRCT)}

Sirveaux et al. [20] have published the largest study so far on the use of the reverse shoulder prosthesis in cuff tear arthropathy. This was a multicentre study involving 80 shoulders in 77 patients with a mean follow-up of 3.6 years. Mean active elevation increased from $73^{\circ}$ to $138^{\circ}$ and the mean Constant increased from 23 pre-operatively to 66 points at follow-up. In $96 \%$ of the shoulders there was no or minimal pain at follow-up. Five cases of glenoid loosening were noted and a scapular notch was seen in $64 \%$ of the shoulders. Better Constant scores were noted if the Teres Minor was intact.

Mulieri et al. [36] reviewed 58 patients (60 shoulders) at a mean of fifty-two months following reverse shoulder arthroplasty for massive rotator cuff tears. 34 shoulders had no previous surgery and 26 patients had a failed previous rotator cuff repair. All patients had improved forward flexion and abduction. Although the external rotation was improved post-operatively this was not statistically significant in both groups (Table 1). In addition a statistically significant improvement in the average American shoulder and Elbow Surgeons Score (ASES) from 33.3 to 75.4, visual analogues score for pain from 6.3 to 1.9 and the visual analogue score for function from 3.2 to 7.1 were noted. $95 \%$ of the patients were satisfied with the outcome, and the implant survival rate was $91 \%$.

Favard et al. [37] retrospectively reviewed 527 arthroplasties. At a minimum follow-up of 5 years in 148 arthroplasties, improved Constant scores as well as forward elevation and external rotation were noted. However, functional results started to decline after 8 years, and increasing frequency of large notches were seen after 5 years. The survivorship rate free of revision was $89 \%$ at 10 years. Guery et al. [34], Cazeneuve and Cristofari [38] have also shown worsening results at longer follow-up. Minimal loosening not seen on radiographs or extension of the cuff tear into the teres minor has been postulated as a cause for this deterioration [34]. Therefore, authors recommend that reverse shoulders should only be offered to patients over 70 years of age.

Reporting on the short term outcomes on the use of reverse shoulder arthroplasty for cuff tear arthropathy in 57 patients, Seebauer et al. [39] noted a $98 \%$ satisfaction rate of the patients. All patients reported almost complete freedom from pain and normal functional outcomes with only slight limitation of internal rotation. A good improvement in function and power can be expected if the deltoid has not been damaged.

\section{Rheumatoid Arthritis}

Reverse shoulder arthroplasty in patients with rheumatoid arthritis is associated with an increased risk of infection and glenoid failure [34]. However, good medium term outcomes have been demonstrated in previous studies [24, 40-44]. In a series of 13 cases with an average follow-up of 87 months, the median Constant score was 59 and there were no infections [43]. However, the authors noted glenoid loosening in 5 of the 13 cases and these deteriorate faster 
Table 1. Short to Medium Term Results of Studies for REVERSE Shoulder Arthroplasty

\begin{tabular}{|c|c|c|c|c|c|c|c|c|c|c|c|c|c|c|c|c|}
\hline \multirow{2}{*}{ 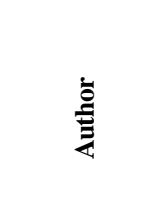 } & \multirow{2}{*}{$\stackrel{\mathscr{J}}{\check{\varpi}}$} & \multirow{2}{*}{ 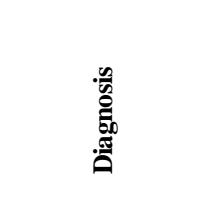 } & \multirow{2}{*}{ 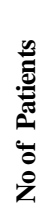 } & \multirow{2}{*}{ 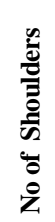 } & \multirow{2}{*}{ 茫 } & \multirow{2}{*}{ 获 } & \multirow{2}{*}{ 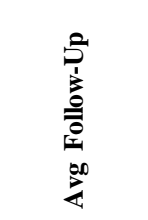 } & \multirow{2}{*}{$\underset{\mathscr{E}}{\ddot{E}}$} & \multicolumn{2}{|c|}{$\begin{array}{c}\text { Mean } \\
\text { Elevation }\end{array}$} & \multicolumn{2}{|c|}{$\begin{array}{c}\text { Mean } \\
\text { Abduction }\end{array}$} & \multicolumn{2}{|c|}{$\begin{array}{c}\text { Mean } \\
\text { External } \\
\text { Rotation }\end{array}$} & \multicolumn{2}{|c|}{$\begin{array}{c}\text { Mean Constant/ } \\
\text { ASES Score }\end{array}$} \\
\hline & & & & & & & & & Pre-op & Post-op & Pre-op & Post-op & Pre-op & Post-op & Pre-op & Post-op \\
\hline Wall [23] & 2007 & $\begin{array}{l}\text { CTA, MRCT, } \\
\text { Fx, REV, T, } \\
\text { RA, FS }\end{array}$ & 186 & 191 & 72.7 & $23-86$ & 39.9 & 24-118 & 86 & 137 & NS & NS & 8 & 6 & 23 & 60 \\
\hline Favard [37] & 2011 & CTA. MRCT, OA & 138 & 148 & 73 & $40-90$ & minimum 60 & NS & 69.3 & 128.6 & NS & NS & 4.9 & 10.6 & 23.9 & 61.5 \\
\hline Guery [34] & 2006 & $\begin{array}{l}\text { MRCT, RA, } \\
\text { FS, REV }\end{array}$ & 77 & 80 & 79.4 & $67-92$ & 69.6 & $60-121$ & NS & NS & NS & NS & NS & NS & NS & NS \\
\hline $\begin{array}{c}\text { Rittmeister } \\
{[24]}\end{array}$ & 2001 & RA & 7 & 8 & 68.8 & $34-86$ & 54.3 & $48-73$ & NS & NS & NS & NS & NS & NS & 17 & 63 \\
\hline Boileau [26] & 2006 & $\begin{array}{l}\text { CTA, MRCT, } \\
\text { FS, REV }\end{array}$ & 45 & 45 & 72 & $50-87$ & 40 & $24-72$ & 82 & 123 & NS & NS & 5 & 7 & 17 & 59 \\
\hline Bufquin [46] & 2007 & $\mathrm{Fx}$ & 43 & 43 & 78 & $65-97$ & 22 & $6-58$ & NS & 97 & NS & 86 & NS & 30 & 16 & 69 \\
\hline Sirveaux [20] & 2004 & CTA & 77 & 80 & 72.8 & $60-86$ & 44.5 & $24-97$ & 73 & 138 & NS & NS & 4 & 11 & 23 & 66 \\
\hline Gohlke [49] & 2007 & REV & 34 & 34 & 68 & $59-82$ & 31.5 & $12-59$ & 48 & 125 & NS & NS & NS & NS & $18 \%^{\wedge}$ & $63 \%^{\wedge}$ \\
\hline Seebauer [39] & 2005 & CTA & 57 & & 70.1 & NS & 18.2 & NS & NS & 145 & NS & NS & NS & NS & NS & 67 \\
\hline Levy [74] & 2007 & REV & 18 & 19 & 72 & $56-83$ & 44 & $24-89$ & 497.7 & 76.1 & 42 & 77.2 & NS & NS & $29.1 *$ & $61.2 *$ \\
\hline Levy [75] & 2007 & REV & 29 & 29 & 69 & $42-80$ & 35 & NS & 38.1 & 72.7 & 34 & 70 & NS & NS & $22.3^{*}$ & $52.1 *$ \\
\hline Frankle [61] & 2006 & $\begin{array}{l}\text { CTA, MRCT, } \\
\text { RA, REV }\end{array}$ & 60 & 60 & 71 & $34-83$ & 33 & $24-68$ & 55 & 105 & 41 & 102 & 12 & 41 & $34.3^{*}$ & $68.2 *$ \\
\hline Werner [31] & 2005 & CTA, REV & 58 & 58 & 68 & $44-84$ & 38 & NS & 42 & 100 & 43 & 90 & 12 & 41 & $29 \% \wedge$ & $64 \%^{\wedge}$ \\
\hline Woodruff [43] & 2003 & RA & 11 & 13 & 64 & $43-72$ & 87 & $60-110$ & NS & NS & NS & NS & NS & NS & NS & 59 \\
\hline De wilde [76] & 2003 & $\mathrm{~T}$ & 13 & 13 & 48 & $26-68$ & 36 & $5-120$ & NS & 105 & NS & NS & NS & NS & NS & 72.5 \\
\hline $\begin{array}{c}\text { Cazeneuve } \\
\text { [45] }\end{array}$ & 2008 & Fx & 25 & 27 & 75 & $58-92$ & 72 & 24-156 & NS & 120 & NS & NS & NS & NS & NS & 59 \\
\hline Cuff [77] & 2008 & REV & 21 & 22 & 67 & $43-83$ & 43 & $25-66$ & 43 & 80 & 36 & 76 & 10.2 & 25.4 & $31.9 *$ & $57.0^{*}$ \\
\hline Cuff [78] & 2008 & $\begin{array}{l}\text { MRCT, } \\
\text { FS, REV }\end{array}$ & 94 & 96 & 72 & $52-88$ & 27.5 & $24-38$ & 64 & 118 & 61 & 110 & 13 & 28 & $30 *$ & $77.6^{*}$ \\
\hline Sayana [79] & 2009 & CTA & 18 & 19 & 73 & $66-80$ & 30 & $18-66$ & NS & NS & NS & NS & NS & NS & 14.8 & 60.9 \\
\hline Grassi [80] & 2009 & $\begin{array}{l}\text { MRCT, CTA, } \\
\text { FS, REV }\end{array}$ & 23 & 23 & 75 & $62-84$ & 42 & $26-84$ & 65 & 133 & NS & NS & 16 & 16 & 22 & 56 \\
\hline Klein [81] & 2010 & $\begin{array}{l}\text { MRCT, } \\
\text { FS, REV }\end{array}$ & 139 & 141 & 72 & $41-87$ & 31 & $24-51$ & 67 & 140 & 65 & 126 & 20 & 49 & $39.1 *$ & $75.1 *$ \\
\hline Mulleri [36] & 2010 & MRCT & 58 & 60 & 71 & $52-88$ & 52 & 24-101 & 53 & 134 & 49 & 125 & 27 & 51 & $33.3^{*}$ & $75.4^{*}$ \\
\hline John [41] & 2010 & RA & 15 & 17 & 67 & $46-85$ & 24 & $12-41$ & 68 & 123 & 66 & 120 & 20 & 53 & 19 & 60 \\
\hline Holcomb [40] & 2010 & RA & 18 & 18 & 72 & $56-86$ & 37 & $34-73$ & 52 & 126 & 55 & 116 & 19 & 22 & $15^{*}$ & $45^{*}$ \\
\hline Young [42] & 2011 & RA & 16 & 18 & 70 & $46-84$ & 44 & $25-84$ & 78 & 139 & 17 & $\mathrm{NS}$ & NS & 46 & 23 & 65 \\
\hline Ekelund [44] & 2011 & RA & 23 & 27 & 68 & $45-80$ & 56 & $18-143$ & 33 & 115 & 26 & 103 & 0.6 & 5.8 & 13 & 52 \\
\hline Nolan [69] & 2011 & CTA & 67 & 71 & 74 & $54-92$ & 24 & $12-58$ & 61 & 121 & NS & NS & 14 & 15 & 28 & 62 \\
\hline Naveed [70] & 2011 & CTA & 43 & 50 & 81 & $59-95$ & 39 & $12-81$ & 55 & 105 & NS & 85 & NS & NS & 17 & 59 \\
\hline Boulahia [63] & 2002 & $\begin{array}{c}\text { CTA, } \\
\text { MRCT, FS }\end{array}$ & 16 & 16 & 72 & $66-80$ & 35 & $24-65$ & 70 & 138 & NS & NS & 6 & 3 & 22 & 59 \\
\hline Patel [82] & 2012 & REV & 28 & 28 & 68 & $68-77$ & 41 & $19-73$ & 44 & 108 & NS & NS & NS & NS & $24^{*}$ & $66^{*}$ \\
\hline
\end{tabular}

*ASES score (American Shoulder and Elbow Surgeons score).

${ }^{\wedge}$ Relative Constant score.

MRCT - Massive rotator cuff tear; CTA - Cuff tear arthropathy; REV - Revision; FX - Fracture; FS - Fracture Sequelae; T - Tumour 
than in rotator cuff disease. Young et al. [42] also did not have any infection in their series of 18 cases. The Constant Score in their study increased from 23 to 65 and forward elevation from 78 to 139 . Although scapular notching was seen in ten of the eighteen shoulders, there were no cases of loosening. According to Holcomb et al. [40], there is no correlation between disease severity and outcome. Ekelund and Nyberg [44], have published the largest study of 27 shoulders with rheumatoid arthritis undergoing reverse shoulder arthroplasty. They found an overall complication rate of $15 \%$, no glenoid or humeral loosening and notching of various degrees were seen in $52 \%$ of the cases. As no long term results are available, most authors recommend caution in this group of patients. Adequate glenoid bone stock is the most important requirement for reverse arthroplasty [17].

\section{Acute Complex Fractures of the Proximal Humerus}

Elderly patients with poor bone quality sustaining a complex three or four part fracture are a treatment challenge and a new indication for reverse shoulder arthroplasty [4547]. Recovery is faster compared to hemiarthroplasty and there is a decreased requirement for rehabilitation [17]. Hemiarthroplasty provides excellent results with tuberosity reattachment and if the tuberosity heals anatomically, but the results are poor in cases of non-union of the greater tuberosity [47].

At a short term follow-up of 22 months in 43 patients, Bufquin et al. [46] demonstrated satisfactory function and range of movement despite tuberosity migration in $53 \%$ and a scapular notch in $25 \%$. Cazeneuve et al. [45] evaluated the results of 27 three and four part fractures at a mean followup of 72 months, and found good results for pain, mediocre for strength and disappointing results for mobility. Although anterior elevation and abduction recovered, internal rotation was limited allowing the hand to rarely reach the sacrum and very limited external rotation allowing overhead elevation. 14 cases had inferior scapular notching. The same patients when evaluated at a mean follow-up of 6.6 years, the Constant scores had declined and scapular notching had worsened [38].

Gallinet et al. [47] compared hemiarthroplasty (17 patients, mean follow-up 16.5 months) with reverse shoulder arthroplasty (16 patients, mean follow-up 12.4 months) in the treatment of complex proximal humerus fractures. Abduction and forward flexion was significantly better in the reverse prosthesis group, while external rotation was significantly better in the hemiarthroplasty group. Patients in the reverse prosthesis group had significantly better Constant scores. They concluded that reverse prosthesis was better in the management of complex fractures in the elderly patients. Although the outcomes seem comparable to hemiarthroplasty, they are less dependent on tuberosity healing and furthermore no long term results are as yet available. Hence caution should be used. Another study [48] showed superior 5 year functional results in the reverse shoulder prosthesis group when compared to hemiarthroplasty.

\section{Revision of Failed Prosthetic Surgery and Fracture Sequelae}

Reverse shoulder arthroplasty done as revision surgery have a high complication rate, and give mediocre results when compared results of primary surgery [23]. Gohlke and Rolf [49] reported on 34 revisions, and although there was improvement in the range of motion, function was related to the extent of soft tissue damage. There was a $24 \%$ complication rate as well. Wall et al. [23] noted that patients undergoing revision surgery had significantly worse range of movement and Constant scores. Furthermore, a risk of complication of $37 \%$ associated with revision surgery was higher than the risk of complication associated with primary surgery of $13 \%$. According to Werner et al. [31] prior operation is a risk factor for postoperative complications. Reoperation rate was $18 \%$ after primary surgery and $39 \%$ after revision surgery. In addition they have reported a complication rate of $50 \%$ in patients undergoing revisions after previous prosthetic surgery. Boileau et al. [26] noted similar results with higher complications in the revision group than the primary group $(47 \%$ vs $5 \%)$. Although functional improvement and better range of motion was noticed after reverse shoulder arthroplasty for proximal humeral non-union, rate of dislocation was high [50].

\section{COMPLICATIONS}

Reverse shoulder prosthesis is a double-edged sword and must be used with caution. High complication rates have been reported in the literature. Revision surgery is associated with a four times increase in risk of complications [35]. Common complications are outlined below.

\section{Infection}

Infection rates are four times that of total shoulder arthroplasty and are thought to be related to the design of implant [17]. It is thought that the inverted design creates a subacromial dead space which leads to haematoma accumulation. Furthermore, the large surface area of the implant can be infected easily. Infection rates of $1-15 \%$ have been reported in the literature [23, 35, 37, 49], with higher rates in revision surgery [31]. Commonest pathogen isolated from infected reverse shoulder implants is Propionibacterium acnes. Coagulse-negative staphylococcus aureus, and methicillin-resistant staphylococcus aureus were found less commonly [51]. Priopionibacterium acnes infections are a challenge to diagnose as they display minimal clinical signs, inflammatory markers are commonly normal and cultures may not be positive for as long as 2 weeks [52].

\section{Scapular Notching}

Inferior scapular neck notching has an incidence varying from $0 \%$ to $96 \%[20,35,53,54]$. This is the result of bony erosion occurring as a result of the humeral component repetitively impinging on the scapular neck [55]. While some studies have shown it to be related to poorer clinical results $[20,55]$, others have found no such association [23, 53]. Favard et al. [37] noted progressive enlarging of the 
scapular notches at long-term follow up but no correlation was noted with the clinical outcome. According to Nyffeler et al. [56], placing the glenosphere inferiorly on the bony glenoid prevents notching. A minute overhang of even $1 \mathrm{~mm}$ has been shown to significantly decrease the incidence of notching [57]. No glenoid notching or loosening was noticed in a new prosthesis design implanted in 76 patients at a mean follow-up of 44 months [54]. This implant had a lateralised centre of rotation by $8 \mathrm{~mm}$. In another study by Boileau $\mathrm{et} \mathrm{al}$. [58] lateralization of the centre of rotation was achieved by using bone graft from the humeral head to create a longer scapular neck, thus lessening impingement of the humerus on the scapula. This technique has been termed Bony Increased Offset Reverse Shoulder Arthroplasty or BIORSA. According to the authors the main advantage is keeping the centre of rotation at the prosthesis-bone interface, which decreases torque on the glenoid component. In a prospective study of 42 patients with mean follow-up of 28 months no glenoid loosening was noted and the notching was observed in only 8 of 42 cases. Although postoperative complications and revision rates decrease with experience, notching has not been shown to decrease with experience [59].

\section{Instability}

Reported dislocation rates in the literature are between 0 and 30\% [20, 22, 31, 60-63]. Wall et al. [23] have reported a $7.5 \%$ dislocation rate in 191 reverse shoulder arthroplasties at a mean follow-up of 39.9 months. In a systematic review by Zumstein et al. [35], instability was the most common complication observed in 782 reverse shoulder arthroplasties, with a mean incidence of $4.7 \%$. The incidence was doubled in patients who had reverse shoulder arthroplasty for revision of previous total shoulder replacement or hemiarthroplasty. $97 \%$ of the shoulders which had instability were operated on by a deltopectoral approach. Several possible causes of instability have been suggested: subscapularis insufficiency, previous trauma causing distortion of the bony and soft tissue anatomy, malposition of the components, poor deltoid tension and the humeral component levering against the glenoid [63, 64].

Favre et al. [61] experimented with the effect of component position in preventing anterior dislocation. They concluded that intrinsic stability is predominantly dependent on version of the humeral component with glenoid version playing only a minor role. They further concluded that the stability can be improved by inserting the humeral component in neutral or slight anteversion. According to Guiterrez et al. [65] stability is mainly achieved by compressive forces generated by muscles with lesser effects achieved from socket depth. Glenosphere size contributes least to the stability of the reverse shoulder.

In a prospective study of 138 patients Edward et al. [66] noted that all dislocations occurred in patients whose subscapularis tendon was irreparable. They concluded that an attempt to repair subscapularis tendon should always be made. Similar conclusions have been drawn from other studies [23,26,31]. Surgical error is likely the cause for dislocations occurring in the first 3 months after implantation and closed reduction is generally not successful. Late dislocations can be treated with a successful closed reduction [17].

Insufficient tension of the deltoid causing instability is difficult to address. Determination of the tension is a "feel" thing and is guided by surgical experience. Boileau et al. [14] have suggested that the conjoint tendon should feel tensioned after reduction. Over tensioning the deltoid can cause stress fractures in an acromion that has already been weakened by the superior migration of humeral head as in cuff arthropathy [14].

\section{Acromial Insufficiency and Fractures}

Superior subluxation of the humeral head causes erosion to the acromion resulting in it being thin and insufficient [67]. Walch et al. [68] noted acromial insufficiency in 41 (9\%) of 457 reverse shoulder arthroplasty implantations. 17 had a fracture of the acromion, 23 had os acomiale and 1 had a pseudoarthrosis of the scapular spine. Their study showed that preoperative acromial pathology did not affect subjective or functional results compared to patients without acromial pathology. They had 4 cases $(0.8 \%)$ of postoperative scapular spine fractures which showed poor results. Post-operative acromial fractures have an incidence between $1.4 \%$ to $4 \%[69,70]$, and occur either through the acromion or at the base of the spine of scapula [68,71]. Although exhibiting little pain, the patients often have sudden deterioration in function [68]. Furthermore, they have reduced range of movement, functional score and subjective satisfaction $[68,70]$.

\section{Nerve Palsy}

A recent study has shown subclinical neurological disturbance in $47 \%$ of the patients undergoing reverse shoulder arthroplasty. Axillary nerve is mainly involved but the deficit is usually transient and is believed to be closely associated to the extent of arm lengthening [72]. Van Hoof $e t$ al. [73] estimated an increase in strain of up to $15 \%$ and $19 \%$ respectively for the lateral and medial roots of the median nerve after insertion of reverse shoulder prosthesis. The increased strain could lead to a permanent neurological deficit. Reverse shoulder replacement is associated with a 10 fold higher risk of nerve injury compared to total shoulder arthroplasty though this is usually transient [72].

\section{CONCLUSION}

Reverse shoulder prosthesis is a relatively new implant. Medium term results so far appear to be promising with high levels of patient satisfaction and function. Survival rate of the implant at 10 years is between $84 \%-93 \%$. Some studies have noticed a decline in the results at 10 years follow-up. However, bigger studies with long term follow-up are lacking. Learning curve for the procedure is between 40 and 60 cases after which a reduction in the complication rate could be expected [83].

\section{CONFLICT OF INTEREST}

The authors confirm that this article content has no conflict of interest.

\section{ACKNOWLEDGEMENTS}

Declared none. 


\section{REFERENCES}

[1] Arntz CT, Jackins S, Matsen FA 3rd. Prosthetic replacement of the shoulder for the treatment of defects in the rotator cuff and the surface of the glenohumeral joint. J Bone Joint Surg Am 1993; 75(4): 485-91.

[2] Pollock RG, Deliz ED, McIlveen SJ, Flatow EL, Bigliani LU. Prosthetic replacement in rotator cuff-deficient shoulders. J Shoulder Elbow Surg 1992; 1(4): 173-86.

[3] Neer CS,2nd, Watson KC, Stanton FJ. Recent experience in total shoulder replacement. J Bone Joint Surg Am 1982; 64(3): 319-37.

[4] Neer CS,2nd, Craig EV, Fukuda H. Cuff-tear arthropathy. J Bone Joint Surg Am 1983; 65(9): 1232-44.

[5] Franklin JL, Barrett WP, Jackins SE, Matsen FA 3rd. Glenoid loosening in total shoulder arthroplasty. Association with rotator cuff deficiency. J Arthroplasty 1988; 3(1): 39-46.

[6] Sanchez-Sotelo J, Cofield RH, Rowland CM. Shoulder hemiarthroplasty for glenohumeral arthritis associated with severe rotator cuff deficiency. J Bone Joint Surg Am 2001; 83-A(12): 1814-22.

[7] Williams GR Jr, Rockwood CA Jr. Hemiarthroplasty in rotator cuff-deficient shoulders. J Shoulder Elbow Surg 1996; 5(5): 362-7.

[8] Wirth MA, Rockwood CA Jr. Complications of total shoulderreplacement arthroplasty. J Bone Joint Surg Am 1996; 78(4): 60316.

[9] Fenlin JM Jr. Total glenohumeral joint replacement. Orthop Clin North Am 1975; 6(2): 565-83.

[10] Brostrom LA, Wallensten R, Olsson E, Anderson D. The Kessel prosthesis in total shoulder arthroplasty. A five-year experience. Clin Orthop Relat Res 1992; (277): 155-60.

[11] Gerard Y. Is a retentive total shoulder prosthesis possible? Acta Orthop Belg 1985; 51(4): 616-24.

[12] Kolbel R, Friedebold G. Shoulder joint replacement. Arch Orthop Unfallchir 1973; 76(1): 31-9.

[13] Wretenberg PF, Wallensten R. The Kessel total shoulder arthroplasty. A 13- to 16-year retrospective followup. Clin Orthop Relat Res 1999; (365): 100-3.

[14] Boileau P, Watkinson DJ, Hatzidakis AM, Balg F. Grammont reverse prosthesis: design, rationale, and biomechanics. J Shoulder Elbow Surg 2005; 14(1 Suppl S): 147S-61S.

[15] Grammont P, Trouilloud P, Laffay J, Deries X. Concept study and realization of a new total shoulder prosthesis. Rhumatologie 1987; 39: 407-18.

[16] Matsen FA 3rd, Boileau P, Walch G, Gerber C, Bicknell RT. The reverse total shoulder arthroplasty. J Bone Joint Surg Am 2007; 89(3): 660-7.

[17] Gerber C, Pennington SD, Nyffeler RW. Reverse total shoulder arthroplasty. J Am Acad Orthop Surg 2009; 17(5): 284-95.

[18] Amstutz HC, Sew Hoy AL, Clarke IC. UCLA anatomic total shoulder arthroplasty. Clin Orthop Relat Res 1981; (155): 7-20.

[19] Amstutz HC, Thomas BJ, Kabo JM, Jinnah RH, Dorey FJ. The Dana total shoulder arthroplasty. J Bone Joint Surg Am 1988; 70(8): 1174-82.

[20] Sirveaux F, Favard L, Oudet D, Huquet D, Walch G, Mole D. Grammont inverted total shoulder arthroplasty in the treatment of glenohumeral osteoarthritis with massive rupture of the cuff. Results of a multicentre study of 80 shoulders. J Bone Joint Surg $\mathrm{Br} 2004$; 86(3): 388-95.

[21] Boileau P, Sinnerton RJ, Chuinard C, Walch G. Arthroplasty of the shoulder. J Bone Joint Surg Br 2006; 88(5): 562-75.

[22] Ecklund KJ, Lee TQ, Tibone J, Gupta R. Rotator cuff tear arthropathy. J Am Acad Orthop Surg 2007; 15(6): 340-9.

[23] Wall B, Nove-Josserand L, O'Connor DP, Edwards TB, Walch G. Reverse total shoulder arthroplasty: a review of results according to etiology. J Bone Joint Surg Am 2007; 89(7): 1476-85.

[24] Rittmeister M, Kerschbaumer F. Grammont reverse total shoulder arthroplasty in patients with rheumatoid arthritis and nonreconstructible rotator cuff lesions. J Shoulder Elbow Surg 2001; 10(1): 17-22.

[25] Martin TG, Iannotti JP. Reverse total shoulder arthroplasty for acute fractures and failed management after proximal humeral fractures. Orthop Clin North Am 2008; 39(4): 451-7, vi.

[26] Boileau P, Watkinson D, Hatzidakis AM, Hovorka I. Neer Award 2005: The Grammont reverse shoulder prosthesis: results in cuff tear arthritis, fracture sequelae, and revision arthroplasty. J Shoulder Elbow Surg 2006; 15(5): 527-40.
De Wilde LF, Plasschaert FS, Audenaert EA, Verdonk RC Functional recovery after a reverse prosthesis for reconstruction of the proximal humerus in tumor surgery. Clin Orthop Relat Res 2005; (430): 156-62.

[28] Mavrogenis AF, Mastorakos DP, Triantafyllopoulos G, Sakellariou VI, Galanis EC, Papagelopoulos PJ. Total scapulectomy and constrained reverse total shoulder reconstruction for a Ewing's sarcoma. J Surg Oncol 2009; 100(7): 611-5.

[29] Holcomb JO, Cuff D, Petersen SA, Pupello DR, Frankle MA. Revision reverse shoulder arthroplasty for glenoid baseplate failure after primary reverse shoulder arthroplasty. J Shoulder Elbow Surg 2009; 18(5): 717-23

[30] Levy JC, Virani N, Pupello D, Frankle M. Use of the reverse shoulder prosthesis for the treatment of failed hemiarthroplasty in patients with glenohumeral arthritis and rotator cuff deficiency. J Bone Joint Surg Br 2007; 89(2): 189-95.

[31] Werner CM, Steinmann PA, Gilbart M, Gerber C. Treatment of painful pseudoparesis due to irreparable rotator cuff dysfunction with the Delta III reverse-ball-and-socket total shoulder prosthesis. J Bone Joint Surg Am 2005; 87(7): 1476-86.

[32] Ackland DC, Roshan-Zamir S, Richardson M, Pandy MG. Moment arms of the shoulder musculature after reverse total shoulder arthroplasty. J Bone Joint Surg Am 2010; 92(5): 1221-30.

[33] Schwartz DG, Kang SH, Lynch TS, et al. The anterior deltoid's importance in reverse shoulder arthroplasty: a cadaveric biomechanical study. J Shoulder Elbow Surg 2013; 22(3): 357-64.

[34] Guery J, Favard L, Sirveaux F, Oudet D, Mole D, Walch G. Reverse total shoulder arthroplasty. Survivorship analysis of eighty replacements followed for five to ten years. J Bone Joint Surg Am 2006; 88(8): 1742-7.

[35] Zumstein MA, Pinedo M, Old J, Boileau P. Problems, complications, reoperations, and revisions in reverse total shoulder arthroplasty: a systematic review. J Shoulder Elbow Surg 2011; 20(1): 146-57.

[36] Mulieri P, Dunning P, Klein S, Pupello D, Frankle M. Reverse shoulder arthroplasty for the treatment of irreparable rotator cuff tear without glenohumeral arthritis. J Bone Joint Surg Am 2010; 92(15): 2544-56.

[37] Favard L, Levigne C, Nerot C, Gerber C, De Wilde L, Mole D. Reverse prostheses in arthropathies with cuff tear: are survivorship and function maintained over time? Clin Orthop Relat Res 2011; 469(9): 2469-75

[38] Cazeneuve JF, Cristofari DJ. The reverse shoulder prosthesis in the treatment of fractures of the proximal humerus in the elderly. $\mathrm{J}$ Bone Joint Surg Br 2010; 92(4): 535-9.

[39] Seebauer L, Walter W, Keyl W. Reverse total shoulder arthroplasty for the treatment of defect arthropathy. Oper Orthop Traumatol 2005; 17(1): $1-24$.

[40] Holcomb JO, Hebert DJ, Mighell MA, et al. Reverse shoulder arthroplasty in patients with rheumatoid arthritis. J Shoulder Elbow Surg 2010; 19(7): 1076-84.

[41] John M, Pap G, Angst F, et al. Short-term results after reversed shoulder arthroplasty (Delta III) in patients with rheumatoid arthritis and irreparable rotator cuff tear. Int Orthop 2010; 34(1): 71-7.

[42] Young AA, Smith MM, Bacle G, Moraga C, Walch G. Early results of reverse shoulder arthroplasty in patients with rheumatoid arthritis. J Bone Joint Surg Am 2011; 93(20): 1915-23.

[43] Woodruff MJ, Cohen AP, Bradley JG. Arthroplasty of the shoulder in rheumatoid arthritis with rotator cuff dysfunction. Int Orthop 2003; 27(1): 7-10.

[44] Ekelund A, Nyberg R. Can reverse shoulder arthroplasty be used with few complications in rheumatoid arthritis? Clin Orthop Relat Res 2011; 469(9): 2483-8.

[45] Cazeneuve J, Hassan Y, Kermad F, Brunel A. Delta III reverseball-and-socket total shoulder prosthesis for acute complex fractures of the proximal humerus in elderly population. Eur J Orthop Surg Traumatol 2008; 18(2): 81-6.

[46] Bufquin T, Hersan A, Hubert L, Massin P. Reverse shoulder arthroplasty for the treatment of three- and four-part fractures of the proximal humerus in the elderly: a prospective review of 43 cases with a short-term follow-up. J Bone Joint Surg Br 2007; 89(4): 516-20.

[47] Gallinet D, Clappaz P, Garbuio P, Tropet Y, Obert L. Three or four parts complex proximal humerus fractures: hemiarthroplasty versus 
reverse prosthesis: a comparative study of 40 cases. Orthop Traumatol Surg Res 2009; 95(1): 48-55.

[48] Boyle MJ, Youn SM, Frampton CM, Ball CM. Functional outcomes of reverse shoulder arthroplasty compared with hemiarthroplasty for acute proximal humeral fractures. J Shoulder Elbow Surg 2013; 22(1): 32-7.

[49] Gohlke F, Rolf O. Revision of failed fracture hemiarthroplasties to reverse total shoulder prosthesis through the transhumeral approach: method incorporating a pectoralis-major-pedicled bone window. Oper Orthop Traumatol 2007; 19(2): 185-208.

[50] Martinez AA, Bejarano C, Carbonel I, Iglesias D, Gil-Alvaroba J, Herrera A. The treatment of proximal humerus nonunions in older patients with the reverse shoulder arthroplasty. Injury 2012; [Epub ahead of print].

[51] Beekman PD, Katusic D, Berghs BM, Karelse A, De Wilde L. One-stage revision for patients with a chronically infected reverse total shoulder replacement. J Bone Joint Surg Br 2010; 92(6): 81722.

[52] Dodson CC, Craig EV, Cordasco FA, et al. Propionibacterium acnes infection after shoulder arthroplasty: a diagnostic challenge. J Shoulder Elbow Surg 2010; 19(2): 303-7.

[53] Levigne C, Boileau P, Favard L, et al. Scapular notching in reverse shoulder arthroplasty. J Shoulder Elbow Surg 2008; 17(6): 925-35.

[54] Valenti P, Sauzieres P, Katz D, Kalouche I, Kilinc AS. Do less medialized reverse shoulder prostheses increase motion and reduce notching? Clin Orthop Relat Res 2011; 469(9): 2550-7.

[55] Simovitch RW, Zumstein MA, Lohri E, Helmy N, Gerber C. Predictors of scapular notching in patients managed with the Delta III reverse total shoulder replacement. J Bone Joint Surg Am 2007; 89(3): 588-600.

[56] Nyffeler RW, Werner CM, Gerber C. Biomechanical relevance of glenoid component positioning in the reverse Delta III total shoulder prosthesis. J Shoulder Elbow Surg 2005; 14(5): 524-8.

[57] de Wilde LF, Poncet D, Middernacht B, Ekelund A. Prosthetic overhang is the most effective way to prevent scapular conflict in a reverse total shoulder prosthesis. Acta Orthop 2010; 81(6): 719-26.

[58] Boileau P, Moineau G, Roussanne Y, O'Shea K. Bony increasedoffset reversed shoulder arthroplasty: minimizing scapular impingement while maximizing glenoid fixation. Clin Orthop Relat Res 2011; 469(9): 2558-67.

[59] Walch G, Bacle G, Ladermann A, Nove-Josserand L, Smithers CJ. Do the indications, results, and complications of reverse shoulder arthroplasty change with surgeon's experience? J Shoulder Elbow Surg 2012; 21(11): 1470-7.

[60] Trappey GJ,4th, O'Connor DP, Edwards TB. What are the instability and infection rates after reverse shoulder arthroplasty? Clin Orthop Relat Res 2011; 469(9): 2505-11.

[61] Frankle M, Siegal S, Pupello D, Saleem A, Mighell M, Vasey M. The Reverse Shoulder Prosthesis for glenohumeral arthritis associated with severe rotator cuff deficiency. A minimum twoyear follow-up study of sixty patients. J Bone Joint Surg Am 2005; 87(8): 1697-705.

[62] Gallo RA, Gamradt SC, Mattern CJ, et al. Instability after reverse total shoulder replacement. J Shoulder Elbow Surg 2011; 20(4): 584-90.

[63] Boulahia A, Edwards TB, Walch G, Baratta RV. Early results of a reverse design prosthesis in the treatment of arthritis of the shoulder in elderly patients with a large rotator cuff tear. Orthopedics 2002; 25(2): 129-33.

[64] Favre P, Sussmann PS, Gerber C. The effect of component positioning on intrinsic stability of the reverse shoulder arthroplasty. J Shoulder Elbow Surg 2010; 19(4): 550-6.
[65] Gutierrez S, Keller TS, Levy JC, Lee WE,3rd, Luo ZP. Hierarchy of stability factors in reverse shoulder arthroplasty. Clin Orthop Relat Res 2008; 466(3): 670-6.

[66] Edwards TB, Williams MD, Labriola JE, Elkousy HA, Gartsman GM, O'Connor DP. Subscapularis insufficiency and the risk of shoulder dislocation after reverse shoulder arthroplasty. J Shoulder Elbow Surg 2009; 18(6): 892-6.

[67] Smith CD, Guyver P, Bunker TD. Indications for reverse shoulder replacement: a systematic review. J Bone Joint Surg Br 2012; 94(5): $577-83$.

[68] Walch G, Mottier F, Wall B, Boileau P, Mole D, Favard L. Acromial insufficiency in reverse shoulder arthroplasties. J Shoulder Elbow Surg 2009; 18(3): 495-502.

[69] Nolan BM, Ankerson E, Wiater JM. Reverse total shoulder arthroplasty improves function in cuff tear arthropathy. Clin Orthop Relat Res 2011; 469(9): 2476-82.

[70] Naveed MA, Kitson J, Bunker TD. The Delta III reverse shoulder replacement for cuff tear arthropathy: a single-centre study of 50 consecutive procedures. J Bone Joint Surg Br 2011; 93(1): 57-61.

[71] Wahlquist TC, Hunt AF, Braman JP. Acromial base fractures after reverse total shoulder arthroplasty: report of five cases. J Shoulder Elbow Surg 2011; 20(7): 1178-83.

[72] Ladermann A, Lubbeke A, Melis B, et al. Prevalence of neurologic lesions after total shoulder arthroplasty. J Bone Joint Surg Am 2011; 93(14): 1288-93.

[73] Van Hoof T, Gomes GT, Audenaert E, Verstraete K, Kerckaert I, D'Herde K. 3D computerized model for measuring strain and displacement of the brachial plexus following placement of reverse shoulder prosthesis. Anat Rec (Hoboken) 2008; 291(9): 1173-85.

[74] Levy JC, Virani N, Pupello D, Frankle M. Use of the reverse shoulder prosthesis for the treatment of failed hemiarthroplasty in patients with glenohumeral arthritis and rotator cuff deficiency. J Bone Joint Surg Br 2007; 89(2): 189-95.

[75] Levy J, Frankle M, Mighell M, Pupello D. The use of the reverse shoulder prosthesis for the treatment of failed hemiarthroplasty for proximal humeral fracture. J Bone Joint Surg Am 2007; 89(2): 292300.

[76] De Wilde L, Sys G, Julien Y, Van Ovost E, Poffyn B, Trouilloud P. The reversed Delta shoulder prosthesis in reconstruction of the proximal humerus after tumour resection. Acta Orthop Belg 2003; 69(6): 495-500.

[77] Cuff DJ, Virani NA, Levy J, et al. The treatment of deep shoulder infection and glenohumeral instability with debridement, reverse shoulder arthroplasty and postoperative antibiotics. J Bone Joint Surg Br 2008; 90(3): 336-42.

[78] Cuff D, Pupello D, Virani N, Levy J, Frankle M. Reverse shoulder arthroplasty for the treatment of rotator cuff deficiency. J Bone Joint Surg Am 2008; 90(6): 1244-51.

[79] Sayana MK, Kakarala G, Bandi S, Wynn-Jones C. Medium term results of reverse total shoulder replacement in patients with rotator cuff arthropathy. Ir J Med Sci 2009; 178(2): 147-50.

[80] Grassi FA, Murena L, Valli F, Alberio R. Six-year experience with the Delta III reverse shoulder prosthesis. J Orthop Surg (Hong Kong) 2009; 17(2): 151-6.

[81] Klein SM, Dunning P, Mulieri P, Pupello D, Downes K, Frankle MA. Effects of acquired glenoid bone defects on surgical technique and clinical outcomes in reverse shoulder arthroplasty. J Bone Joint Surg Am 2010; 92(5): 1144-54.

[82] Patel DN, Young B, Onyekwelu I, Zuckerman JD, Kwon YW. Reverse total shoulder arthroplasty for failed shoulder arthroplasty. J Shoulder Elbow Surg 2012; 21(11): 1478-83.

[83] Kempton LB, Ankerson E, Wiater JM. A complication-based learning curve from 200 reverse shoulder arthroplasties. Clin Orthop Relat Res 2011; 469(9): 2496-504. 